\title{
Training in Gastrointestinal Motility
}

\author{
Henry P. Parkman \\ Gastroenterology Section, Department of Medicine, Temple University School of Medicine, \\ Philadelphia, Pa., USA
}

\author{
Key Words \\ Gastrointestinal motility · Gastroenterology fellows • \\ Fellowship training
}

\begin{abstract}
Gastrointestinal (GI) motility and functional GI disorders are common reasons for patients to see gastroenterologists. Knowledge of the evaluation and treatment of these disorders is important to appropriately care for these patients in clinical practice. Training in GI motility is important to Gl fellows and their subsequent role as gastroenterologists. The aim of this paper is to discuss the importance of GI motility disorders for trainees in gastroenterology, provide some suggestions for training activities for Gl fellows in GI motility, and discuss ways to address the unmet clinical need for caring for patients with GI motility disorders that gastroenterologists routinely see in their clinical practice.
\end{abstract}

Copyright $\subset 2006$ S. Karger AG, Basel

Gastrointestinal (GI) motility and functional GI disorders are the most common reasons for patients to see gastroenterologists. Knowledge of the evaluation and treatment of these disorders is important to appropriately care for these patients in clinical practice. Paradoxically, the time GI fellows spend learning about these disorders during their training is relatively low. This paper discusses the importance of GI motility disorders for trainees in gastroenterology and some suggestions for training activities for GI fellows so they are equipped to treat these types of patients that they will routinely see in their clinical practice after training.

\section{Importance of GI Motility Disorders}

GI motility and functional bowel disorders, such achalasia, gastroesophageal reflux disease, gastroparesis, functional dyspepsia, irritable bowel syndrome, colonic inertia, pelvic floor dyssynergia, and fecal incontinence, affect up to $25 \%$ of the US population. These disorders comprise up to $50 \%$ of GI problems for which patients seek health care. GI motility disorders pose a heavy burden of illness, decreased quality of life, and decreased work productivity. Unfortunately, these disorders are often ignored or sidelined because of a lack of understanding of mechanisms and appropriate therapy. Patients with motility disorders can be complex and difficult to treat. Understanding the GI motility dysfunction underpins the appropriate management of the patients.

GI dysmotility also impacts on the quality of life of patients with other disorders. For example, a significant percentage of patients with diabetes have GI dysmotility. GI complications of diabetes can affect one or more parts of the gut and produce nausea, vomiting, abdominal pain, constipation and/or diarrhea. Abnormal gastric emptying, or gastroparesis, may lead to poor glucose control and complications of diabetes. Likewise, esophageal and GI

\section{KARGER \\ Fax +4161306 1234 E-Mail karger@karger.ch} www.karger.com 
motor dysfunction is often present in Parkinson's disease and may lead to trouble swallowing or evacuating the bowels.

GI motility also plays an important role in issues outside of what is traditionally considered gastroenterology. Examples of this include nutrition, obesity, and drug delivery. Nutrition depends on the controlled delivery of food for optimal assimilation from the GI tract. Signaling of satiety is dependent on proper control of GI motility and release of GI hormones; obesity can result when satiety and GI motility are altered. Bioavailability of orally administered drugs is controlled in large part by GI motility.

\section{Current Training Recommendations for Gastroenterology Fellows in GI Motility}

The Gastroenterology Core Curriculum for the training of gastroenterology fellows was initially proposed in 1996 and revised in 2003. In the most recent 2003 version [1], two training levels in GI motility were recommended for gastroenterology fellows.

Level 1 is basic training in GI motility expected of all GI fellows. GI fellows should understand the pathophysiology of GI motility and functional GI disorders, learn to manage patients with these disorders, and understand the usefulness of the various GI motility tests available to evaluate patients. Trainees should have an appropriate clinical outpatient experience in which to see and manage patients with possible motility disorders. GI fellows should learn about the various GI motility tests including the appropriate indications, interpretation of test results, and appropriate treatment of patients. Trainees should have the opportunity for hands-on experience with GI motility studies, including 24-hour esophageal $\mathrm{pH}$ monitoring.

Level 2 training is for trainees who wish to have a subspecialty in GI motility and perform motility studies as consultants to other physicians. These subspecialty trainees should become familiar with performance and interpretation of a number of different types of GI motility tests. The recommended numbers of studies to achieve expertise was suggested to be 50 esophageal manometry studies, 25 esophageal $\mathrm{pH}$ recordings, and 30 anorectal manometry studies. These trainees should also spend at least 3 months in a GI motility laboratory under the preceptorship of experienced clinicians in the performance of these studies.

\section{Current Situation regarding Training and Careers in GI Motility}

With the marked increase in GI endoscopic procedures being performed, there appears to have been a decrease in time for training in GI motility despite lengthening the GI fellowship training to 3 years and the core curriculum recommendations. The number of endoscopic procedures performed by GI fellows often inversely reflects time devoted to GI motility during GI fellowship. In a 1999 survey of recently graduated GI fellows, the median number of endoscopies performed was 500, the median number of colonoscopies performed was 394, yet the median number of esophageal manometries performed was 5, with nearly $40 \%$ of GI fellows doing none [2]. From the fellows' viewpoint, there has been more emphasis on productivity in procedures and consultations than on education and research [2].

Although not carefully documented, there appears to have been a decrease in the number of GI fellows choosing GI motility as a career. This is occurring at a time when there has also been a decrease in interest of GI fellows pursuing a career at academic centers. This decline is due to a multitude of reasons including concerns about lifestyle and salary [3]. Since GI motility specialists are often at academic centers, this may be one of the reasons leading to the decrease in the number of specialists entering this clinically important field.

Career pathways, including interests in GI motility, are fostered early in the training of physicians. The training of medical students, residents, and first year GI fellows is concentrated on hospitalized patients [4], whereas GI motility is generally an outpatient practice. This limits trainee's exposure to patients with GI motility problems and hinders the development of interest in GI motility. Mentorship and role modeling are considered to be important factors influencing a student's career choice $[5,6]$. By the end of their first year of training, at a time when decisions are often made about research plans and career pathways, GI fellows have not often been exposed to GI motility.

Interestingly, GI motility gives rise to a variety of important teaching topics as examples of GI physiology and pathophysiology. A recent editorial by Rodger Liddle discussed the challenges faced in training future gastroenterologists considering the many diverse interests of GI fellows including basic science research, clinical research, and clinical practice [7]. Discussion of GI physiology on teaching rounds, with an emphasis on the physiologic importance and pathophysiologic basis of the diseases, helps bring together the varied interests of fellows and attendings. 


\section{Examples of Different Types of Training Activities in GI Motility}

Some selected aspects will be described which have been instituted at the author's institution to expose trainees to GI motility which has faculty interested in a range of GI motility disorders including the esophagus, stomach, small intestine, colon, and biliary tree/gallbladder. Many of the different GI motility procedures needed for patient evaluation are performed.

\section{Medical Student Clinical Correlation Lectures in GI Motility Disorders}

The medical students of Temple University have a variety of exposures to GI motility. In the first year, a GI physiologist with expertise in GI motility gives the GI physiology lectures. In a separate clinical correlation lecture, a patient with the classic esophageal motility disorder, achalasia, is discussed allowing the students to review normal esophageal motility and understand how abnormal motility can give rise to symptoms. During this interactive clinical correlation session, clinical questions which are based on basic concepts of GI physiology are posed to the students, in order to enhance the understanding and interest in GI motility [8].

\section{Housestaff Research Projects in GI Motility}

Medical housestaff, primarily second and third year residents, are encouraged to perform research projects with attendings. Annually, one morning is set aside which is devoted to presenting the housestaff with research opportunities that are available. Several attendings interested in GI motility present possible projects for interested interns and residents. At the end of the year, the residents present their research projects in oral abstract presentations.

\section{GI Fellow GI Motility Training}

During the 'Pathophysiology Conference' held on Thursday mornings, lectures in GI motility and functional bowel disorders are given. During the 'Endoscopy Conference' held Friday mornings, the director of the GI motility laboratory gives lectures on esophageal manometry, esophageal $\mathrm{pH}$ monitoring, and anal manometry and how these tests contribute to patient management. These lectures help provide the foundation for their patient care that they provide in the GI fellows clinic. The second and third year GI fellows can elect to rotate in the GI motility laboratory where they observe the procedures and review the recordings with the GI motility attending. These activities satisfy the level 1 basic training in GI motility for GI fellows.

\section{Swallowing Conference}

A Dysphagia/Swallowing conference is held monthly. This is a multidisciplinary conference which is run by our GI section and involves participation of GI faculty and GI fellows along with speech pathologists, ENT physicians, surgeons, and radiologists. Generally, a case is discussed with display of the patient's diagnostic tests. Often the care of the patients presented is ongoing and treatment suggestions made at the conference are instituted. Presentations are also given by speech pathologists, radiologists, ENT surgeons, as well as gastroenterologists discussing the tests that they perform to evaluate patients.

\section{GI Motility Research Conference}

A GI Motility Research Conference is held biweekly for individuals interested in GI motility. Participating in the conferences are interested gastroenterologists, physiologists, pharmacologists, radiologists, and pathologists. Fellows performing research in related GI motility areas attend. The objectives of this conference are to present potential areas of research collaboration, current ongoing studies and/or recently completed research projects with the goals of: (1) increasing awareness of research related to GI motility at our institution; (2) discussing areas that new research studies might develop, and (3) encouraging extramural grant applications.

\section{GI Fellow Rotation in Complex GERD and \\ Esophageal Motility Disorders}

Each month, a GI fellow, generally one that is on a research rotation, spends a morning session once a week seeing patients with a specialty attending interested in complex gastroesophageal reflux disease (GERD) and/or esophageal motility problems. The rotation is designed so that the initial evaluation, procedures, review of the tracings, and treatment of the patients are handled by the same fellow, allowing continuity of care for the fellow and the patient. This GI fellow also rotates into the operating room to observe several surgical procedures for GI motility including Heller myotomy, Nissen fundoplication, placement of jejunostomy tube, and placement of gastric stimulator.

\section{GI Motility Fellowship Position}

Our institution has a GI motility fellowship program. This is a specialized 1-year training position for physicians who have either completed a general GI fellowship 
and want to specialize in GI motility or, more commonly, for a physician who has completed housestaff training and wants to do a research year prior to starting their GI fellowship. These GI motility fellows have two main duties: (1) to help oversee the clinical GI motility laboratory by performing and interpreting clinical motility tests, and (2) to perform research involving GI motility. These fellows help perform the electrogastrography (EGG), place catheters for antroduodenal manometry, and perform the anal manometry procedures. They help interpret the clinical tests performed in the motility laboratory, which include esophageal manometry, 24-hour esophageal pH monitoring, anorectal manometry/EMG, antroduodenal manometry, and EGG. The GI motility laboratory performs approximately 240 esophageal manometries and 220 esophageal $\mathrm{pH}$ monitoring studies each year. Formal motility reading sessions are held twice a week allowing tracings to be reviewed by a faculty mentor with the GI motility fellow. At the end of the year, the GI motility fellow is fully trained in a wide range of GI motility tests and generally has completed one or more research projects. These GI motility fellows satisfy the level 2 core curriculum training to be specialists in GI motility.

\section{GI Fellows Research Projects}

Second and third year GI fellows are expected to perform research projects. They are provided research time during their second and third years with designated 3month research blocks. The objectives of the research experience for our GI fellows are to learn about clinical research by setting up, implementing, and completing a research project under the preceptorship of one of the GI faculties. At the end of the academic year, the fellows are expected to present the results of their research projects in a formal presentation and to write a manuscript on their research project. Due to the expertise of our faculty, many of the GI fellows' research projects are often related to GI motility.

\section{The American Motility Society and Trainees in GI Motility}

The American Motility Society (AMS) is the national GI motility organization which seeks to foster excellence in research and medical practice and to promote dissemination of information about the medical conditions encompassed by neurogastroenterology and GI motility. Initially, the function of the AMS was served by holding biennial scientific meetings. These meetings bring together established researchers in GI motility with interested trainees and young investigators. In recent years, the AMS has been doing much more for trainees.

The AMS gives courses on GI motility in clinical practice for individuals at each level of interest in learning about GI motility - GI fellows, practicing physicians, and nurses/technicians that perform the GI motility procedures. These courses are presented by experts actively practicing in the field of GI motility and emphasize new developments in the diagnosis, evaluation and treatment of motility disorders. The goal of these courses is to familiarize and update participants on the current indications, methodology, and interpretation of clinical GI motility tests. In addition, the courses provide in-depth discussion of the physiology and pathophysiology and treatment of GI motility and functional bowel disorders. Multiple formats are used including didactic lectures, workshop discussions and case-based presentations. For each course, the AMS has provided stipends allowing 60 GI fellows to attend free of charge - paying for their room, board, and registration fees. Each meeting has 'Meet the Professor' lunch tables allowing interaction between trainees and the faculty.

Conferences for young investigators interested in a research career in GI motility and neurogastroenterology are held at the biennial scientific meetings to help foster and assist young people starting their research career. During the 2005 AMS meeting, principal investigators shared their experiences in starting up a new laboratory and what they wished they had known before taking their job. This was followed by presentations on how the National Institutes of Health (NIH) system works and how it can work for them.

Recognizing that proper performance of GI motility tests is critically important in the evaluation of patients, the Clinical Practice Committee of the AMS defined standards of practice for a number of clinical motility tests. These guidelines help to better standardize GI motility procedures and are useful not only for motility laboratories, but also for trainees to better appreciate the tests being performed. To supplement these procedure guidelines, patient information sheets have been developed for several GI motility procedures which can be used for distribution to patients who are undergoing a GI motility procedure. These are available through the AMS website at www.motilitysociety.org.

The AMS has been striving for improved coding and reimbursement for procedures performed in the GI motility laboratory. This is important for the proper perfor- 
mance, interpretation and utilization of motility tests in a dedicated motility laboratory in clinical practice. GI motility laboratories are often overseen by a faculty person who is a clinician-investigator who wants to devote their time on GI motility including the care of GI motility patients and training of GI fellows in GI motility. Proper reimbursement is needed to maintain this oversight by a dedicated physician concentrating on motility.

\section{What Are Other Organizations Doing for Trainees?}

Other subspecialties in gastroenterology have invested time and money into training fellows interested to become specialists in their field. Each of the major societies in gastroenterology - the American Gastroenterological Association (AGA), American College of Gastroenterology (ACG), and American Society for Gastrointestinal Endoscopy (ASGE) - have young investigator awards and research awards available for interested, promising individuals. The Functional Bowel Group (FBG) offers 1year research awards in areas related to functional GI disorders. The Crohn's and Colitis Foundation of America (CCFA) has recently started an IBD Fellowship program offering 1-month rotations at identified IBD Centers of Excellence for GI fellows interested in advanced training in inflammatory bowel disease. Advanced 1-year training fellowships are given in the areas of endoscopy and hepatology.

The NIH encourages clinical research by supporting career development awards for junior physician-scientists. The NIH does this by offering training grants to fellows (T32 and F32s) as well as career grants to young faculty for basic science (K08) and clinical research (K23). The Mentored Patient-Oriented Research Career Development Award (K23) is aimed at physicians who have recently completed their clinical training and are at the beginning of their research careers. The 3- to 5-year award requires a $75 \%$ protected time commitment, a research project involving patients, a career development plan, and one or more mentors. Early career physicians who are interested in further career development in biomedical research that is not patient-oriented should consider the Mentored Clinical Scientist Career Development (K08) Award.

In an effort to increase the expertise in clinical research and translational sciences, the NIH has targeted efforts at a variety of trainee levels, not only young attendings, but fellows, residents, medical students, and even those still in high school. In a similar manner, interest in GI disorders and GI motility needs to be fostered at the beginning of training - in medical school and housestaff training years.

\section{GI Fellow Training in GI Motility and Functional GI Disorders}

Training in GI motility is important to GI fellows and their subsequent role as gastroenterologists. This rests on two basic concepts. First, GI motility disorders are important in the health of the general population. Patients with GI motility and functional GI disorders are the types of patients that the GI trainees will be seeing in their clinical practice after training. Second, the evaluation of GI motility and understanding GI motility disorders are important in the care of individualized patients.

For patients with possible GI motility problems, there has been a pendulum swaying between the decision to treat first versus an evaluation first - one that is often decided by the availability of treatments with few side effects versus the ease of the test availability and patient discomfort from the test. For instance, although esophageal manometry, esophageal $\mathrm{pH}$ monitoring, and other esophageal function tests are available, in the last decade with proton pump inhibitors (PPIs), patient evaluation for GERD and esophageal motility disorders is generally performed after a therapeutic trial with a PPI and is reserved for patients that do not fully respond to treatment. Even with the practice of reserving motility testing to nonresponders to empiric therapy, there remain a large number of patients needing evaluation. Appropriately, there appears to be an increasing interest in many private practice groups who are starting to perform motility procedures and an apparent increase in positions advertised for gastroenterologists with GI motility training. Physicians skilled in taking care of these patients, running esophageal/anorectal motility laboratories and knowledgeable about motility disorders often do very well in practice.

GI motility testing has several functions: (1) allowing for the assessment of GI physiology and identification of abnormal physiology and patterns; (2) providing the correct diagnosis of GI motility disorders; (3) guiding treatment of patients, and (4) providing prognostic information for patients (table 1). Some examples of the importance of motility testing in the diagnosis and guiding treatment are readily apparent in clinical practice. For example, esophageal manometry can specifically diag- 
Table 1. Goals of GI motility testing

Allow assessment of GI physiology

Identify abnormal physiology and patterns

Diagnose GI motility disorders

Guide treatment of patients

Provide prognostic information for patients

Table 2. Addressing the unmet clinical need for GI motility

\begin{tabular}{l} 
Increasing awareness of GI motility \\
Importance of GI motility disorders \\
GI motility tests using novel technology \\
New treatments available \\
\hline Training/mentoring in GI motility \\
Reach out to trainees early in their training \\
Increase the number of mentors in GI motility \\
Develop centers of excellence in GI motility \\
Patient evaluation \\
GI motility training \\
Clinical courses in GI motility disorders \\
Promoting GI motility \\
Highlight new concepts in GI motility \\
Promote research in GI motility \\
Increase attractiveness of career opportunities in GI motility \\
Research funding \\
Reimbursement for GI motility tests
\end{tabular}

nose achalasia and provide the rationale for effective, yet invasive, treatments. Anal manometry can determine that the cause of constipation is pelvic floor dyssynergia for which biofeedback may be recommended. Anal manometry biofeedback is also helpful for fecal incontinence.

The GI motility specialist can fully evaluate and care for the patients with GI motility disorders. Often, the GI motility specialist is considered the physician that can take care of the complex GI motility patient that no one else can. Care of patients is becoming more advanced with increasing number of treatments and increasing number of tests for evaluation. For GERD, there has been an increase in the laparoscopic surgical and endoscopic antireflux procedures. Proper selection of patients is needed to help decide on medical treatment, surgical treatment, endoscopic treatment, or no treatment at all. This selection of patients is most often reliably performed with esophageal manometry and esophageal $\mathrm{pH}$ monitoring. This need, in part, may explain the apparent increase in positions advertised for gastroenterologists with GI motility training. This is occurring at a time when coding and billing, and hopefully reimbursement, for GI motility tests have been improving [9]. However, at some centers, in part due to the lack of gastroenterologists with GI motility training, surgeons direct the GI motility laboratory. This is an example of an area of unmet clinical need, namely the need for gastroenterologists trained in GI motility.

\section{Suggestions to Address the Unmet Clinical Need for GI Motility}

Table 2 lists several suggestions to improve trainee interest in GI motility in order to address the current unmet clinical need for caring for patients with GI motility and functional GI disorders. There are three main areas: (1) increasing awareness of GI motility; (2) training/mentoring in GI motility, and (3) promoting GI motility. Increasing awareness of the importance of GI motility disorders will help GI fellows understand that this training is needed for their clinical practice [1]. For didactic teaching, updated teaching modules for the instruction of GI motility and its disorders have been developed [10, 11]. These teaching modules should be further developed to demonstrate innovations in GI motility with the use of video clips and multimedia presentations. Awareness is needed about the variety of GI motility tests available for patient evaluation which allow diagnosis of the patients' condition and help point to the correct treatment. New technologies have recently increased the excitement in evaluation of patients for GI motility disorders since these provide more information and/or increase patient acceptance. Recent examples of using new technology in clinical evaluation include wireless esophageal $\mathrm{pH}$ monitoring with Bravo capsule, impedance monitoring to complement esophageal manometry and esophageal $\mathrm{pH}$ monitoring, and high-resolution contour mapping for esophageal manometry. These newer systems allow improved understanding for the trainee.

To interest individuals in the field of GI motility, reaching out to trainees early in their training is important. In addition to GI fellows, this reaching out should be directed to individuals in medical school and residency training programs. Recruiting interest in GI motility can be strengthened by increasing the number of effective mentors in GI motility - clinicians, clinical investigators, and basic scientists. The NIH has developed grants aimed at clinical scientists interested in mentoring younger po- 
tential investigators. These Midcareer Investigator Award in Patient-Oriented Research (K24) awards are geared to individuals who have a strong track record of performing patient-oriented research and mentoring potential clinical researchers. This award provides salary for $25-50 \%$ effort to continue, and to expand, ongoing research and mentoring activities.

Increasing the number of GI motility centers will make more programs available at which trainees can spend time to learn from the experts. These centers can provide mentorship programs allowing GI fellows, practicing gastroenterologists, and/or nurses/technicians to learn and see the procedures needed for patient evaluation. These centers could provide advanced training in some of the specialized procedures performed in GI motility. There are several programs considered to have expertise in GI motility; however, these are limited in number and in their geographic distribution.

Clinical courses in GI motility and functional GI disorders are given by the AMS and other organizations. In addition to their teaching value, these meetings provide interaction between trainees and established investigators. These courses could be further enhanced by 'live' patients undergoing GI motility evaluation.

Promoting the exciting research that has advanced this field, not only in the basic science laboratory but also in the clinical setting, will serve to enhance interest in GI motility. For example, in the basic sciences, ongoing research is helping understand the role of interstitial cells of Cajal as the pacemaker cells of the GI tract. Studying neural-immune interactions is allowing better understanding of the effects of inflammation on GI motility and sensation. Novel methods of imaging and recording techniques are being used in the clinical evaluation of patients to measure GI motility including SPECT imaging, MRI, capsule telemetry, and endoluminal ultrasonography. These new techniques have the ability to provide clearer diagnostic measures that will hopefully allow better understanding of the patient's cause of their symptoms. New concepts have emerged over the last decade in both GI motility and functional GI disorders allowing improved treatment. Electrical stimulation is being used to treat gastroparesis and is being applied to other areas of the luminal GI tract. Neurotoxins are being used locally to abolish excitatory nerve function and treat spastic disorders of the GI tract including achalasia and gastroparesis. As in many areas, our understanding of the physiology has surpassed our current ability to treat many of the pathophysiologic disorders. Current and future clinical research will continue to enhance our armamentarium for treating patients with GI motility disorders [12]. For example, research is ongoing in neural stem cell therapy to replenish the enteric nervous system and treat GI motility disorders.

\section{References}

$\checkmark 1$ AASLD, ACG, AGA, ASGE: Training the gastroenterologists of the future: the Gastroenterology Core Curriculum. Gastroenterology 2003; $124: 1055-1104$.

-2 Hosseini M, Lee JG, Romano P, Hosseini S, Leung $\mathrm{J}$ : Educational experiences and quality of life of gastroenterology fellows in the United States. Am J Gastroenterol 1999;94:36013612.

3 McCashland TM, Zetterman RK, Ruby EI, McCashland CR, Wigton RS: Attitudes and expectations of 1995 gastroenterology graduates about gastroenterology. Am J Gastroenterol 1996;91:2091-2095.

-4 Kirsch M: GI fellowship training - the missing piece. Am J Gastroenterol 2005;100:19121913.

Training in Gastrointestinal Motility
-5 Skeff KM, Mutha S: Role models - guiding the future of medicine. N Engl J Med 1998;339: 2015-2017.

6 Foxx-Orenstein A: Why aren't women attracted to gastroenterology? Am J Gastroenterol 2004;98:190-191.

7 Liddle RA: A unifying principle in training gastroenterologists. Gastroenterology 2004; 127 : 377.

8 Crass MF 3rd, Hodges DS: A novel interactive demonstration in clinical gastroenterology for first-year medical students. Am J Physiol 1993; 264:S1-S3.

-9 Botoman VA, Rao S, Dunlap P, Abell T, Falk GW: Bill Coding and RVS Committee of the American Motility Society. Motility and GI function studies billing and coding guidelines: a position paper of the American Motility Society. Am J Gastroenterol 2003;98:12281236.
10 Brookes SJ, Cook IJ, Costa M, de Giorgio R, Dent J, Grundy D, Sander KM, Schemann M, Shea-Donohue T, Smith TK, Tonina M: Education project for pathophysiology of gastrointestinal motility. Neurogastroenterol Motil 2005;17(suppl 3):2-3.

11 Costa M, Sanders KM, Schemann M, Smith TK, Cook IJ, de Giorgio R, Dent J, Grundy D, Shea-Donohue T, Tonini M, Brookes SJ: A teaching module on cellular control of small intestinal motility. Neurogastroenterol Motil 2005;17(suppl 3):4-19.

12 Micci MA, Kahrig KM, Simmons RS, Sarna SK, Espejo-Navarro MR, Pasricha PJ: Neural stem cell transplantation in the stomach rescues gastric function in neuronal nitric oxide synthase-deficient mice. Gastroenterology 2005;129:1817-1824. 